\title{
INNOVATION OF THE PELVIS TILT FUNCTIONAL DIAGNOSTICS IN CHILDREN
}

\author{
R. Tasheva*, K. Kolev \\ Physical Therapy Department - Theory and Methodology of the Physical Therapy National Sports \\ Academy "Vassil Levski"- Sofia, Bulgaria
}

\begin{abstract}
THE AIM of the research is to make literature review and to study the possibilities for implementation of Digital Pelvic Inclinometer in functional diagnostic of the pelvis inclination during the prevention of the postural disorders in children. METHOD: The Digital Pelvic Inclinometer (DPI) developed by Sub-4 Technologies, is a precision measuring device used to quantify innominate bone inclination. Using the described technique, the DPI can be used to assess pelvic behavior by using the specialize protocols. DPI uses a digital display. This display allows the physiotherapist to see the output of the device while performing the measurement procedure. RESULTS: Twenty-five children were assessed by the DPI. The results show that the pelvic inclination in children were average for left $10.78^{\circ}$ and for right $10.28^{\circ}$. Torsion was the difference between left and right inclination of innominate bones. Mean value of torsion is $2.108^{\circ}$ this indicate potential for developing progression lordosis in children from 1 to 3 class. CONCLUSION This new and innovative methodology allows asymmetry, dysfunction and adaption to be quantified, allowing instant evidence based diagnosis and treatment. The DPI subsequently allows the physiotherapist to record normative values after treatment interventions have been implemented.
\end{abstract}

Key words: Digital Pelvic Inclinometer, pelvic tilt, physiotherapy.

\section{INTRODUCTION}

Traditionally, pelvic tilt has often been measured in clinical practice to identify the presence of abnormal postures that may cause dysfunction and lead to chronic musculoskeletal pain conditions, such as low back pain (1).

The postural disorders in children are a widespread problem. To precise the functional diagnostic in children is the most important part of the prevention. The key point in this aspect is the utilization of the devices. Unfortunately, the literature and the results on the problem are very scant.

THE AIM of the research is to make literature revue and to study the possibilities for

\footnotetext{
*Correspondence to: Rumiana Tasheva, National Sports Academy "Vassil Levski”- Sofia, Bulgaria, Tel: 08933965 83; e-mail: rumiana_tasheva@yahoo.com
}

implementation of Digital Pelvic Inclinometer in functional diagnostic of the pelvis inclination during the prevention of the postural disorders in children.

\section{METHODS}

Different models of caliper - based inclinometer have been investigated in the literature. The Palpation Meter (PALM, Performance Attainment Associates, St. Paul, MN, USA) is the caliper-based inclinometer that has been extensively explored $(1,3-5,7-$ 9). Other models that have been investigated include those developed and modified by Walker (1987) and Crowell (1994) $(2,11)$. The included a spirit level to permit readings relative to the ground, finger-tip rings to allow superior palpation of the bony prominences, and a digital read-out for ease and speed of reading the output (2). The Digital Pelvic Inclinometer (DPI, Sub-4 Limited, UK) is a new, commercially-available, caliper -based inclinometer that is very similar to the model 
developed by Crowell (1994). Like this model the DPI uses a digital display (2). This display allows the physiotherapist to see the output of the device while performing the measurement procedure. In addition, the DPI also has recessed calliper ends, which allow simultaneous palpation of the bony prominences with the hands and the calliper arms. Finally, the DPI also contains a spirit level to facilitate measurements of pelvic angles relative to the ground as well as relative to the other side of the pelvis.
TASHEVA R., et al. The Digital Pelvic Inclinometer (DPI) developed by Sub-4 Technologies, is a precision measuring device used to quantify innominate bone inclination. Using the described technique, the DPI can be used to assess pelvic behavior by using the specialize protocols. The Digital Pelvic Inclinometer (DPI) allows the physiotherapists to understand and measure the role of the pelvic in development of repetitive injury including lower back (Figure 1).

\section{THE DEVICE}

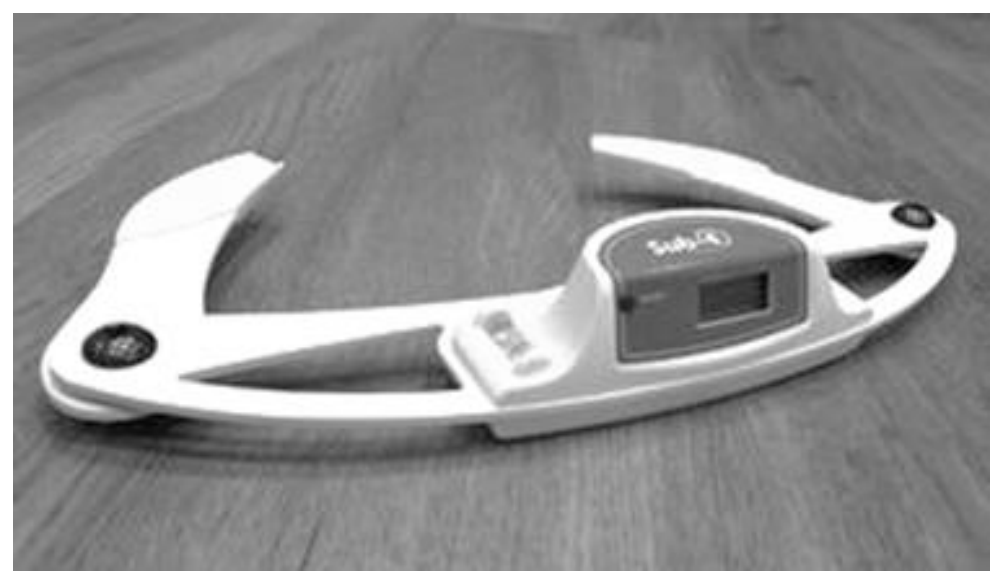

Figure 1. Digital Pelvic Inclinometer

Data is captured by the DPI via a tiny threeaxis accelerometer attached to a small electronics board. The accelerometer measures the static acceleration of gravity in the three directions. The electronics use this data to calculate the angle of tilt. This data is then shown in numerical form on the LCD.

The DPI is only as accurate as it's user and has high inter/intra physiotherapists reliability if used in the correct manner, following the guidelines below. The anterior superior iliac spine (ASIS) and pubic symphysis are aligned vertically. In this position the pelvis is in 'neutral' and is described by Kendall (2005) "as an ideal position for the pelvis" (6). Kendall also describes the normal sagittal plane inclination between the posterior superior iliac spine (PSIS) and ASIS as being approximately $5^{\circ}+\mathrm{ve}$ (Innominate inclination is considered +ve when the PSIS is higher than the ASIS, and -ve when the PSIS is lower than the ASIS). Others have described average inclination at around $11.3^{\circ}+\mathrm{ve}$ and $8.8^{\circ}+\mathrm{ve}$ $3,5.2^{\circ}(6)$.

However, variations as much as twelve degrees have been found in cadaver studies. This author describes the normal inclination of the average adult innominate to be around 8 to $10^{\circ}$ $+\mathrm{ve}$, based on clinical experience.

- The DPI is an easy to use hand-held instrument designed to allow the physiotherapists to measure innominate inclination and establish pelvic torsion.

- The two moveable arms with sensory finger grips increase proprioception and therefore accurate measuring.

- The precision arms pivot about a main body, which houses a digital inclinometer and a spirit level. The spirit level allows the main body to be kept level during measurement.

- The DPI allows the physiotherapists to record quantitative data both before and after treatment (6).

\section{THE PROTOCOLS}

1. Basic Pelvic Torsion MeasurementStart on one side of your patient and take an innominate bone inclination. Record this data.

Then repeat the innominate bone inclination measurement, but this time on the contralateral side. Any difference in the values between each side is described as a pelvic torsion, which may require corrective treatment (Table 1). 
Table 1. An example of a basic pelvic torsion measurement

\begin{tabular}{|l|c|}
\hline Right Innominate Inclination & $15^{0}$ \\
\hline Left innominate Inclination & $8^{0}$ \\
\hline Pelvic Torsion & $7^{0}$ \\
\hline
\end{tabular}

TASHEVA R., et al.

2. The Pelvic Equilibrium Theory

The Pelvic Equilibrium Theory' is an exciting new explanation of pelvic adaption in the presence of leg length inequality (LLI). Discovered by specialist podiatrist Clifton Bradeley this new theory is fast becoming the most popular MSK assessment protocol used in clinical biomechanics across the world (Figure 2).

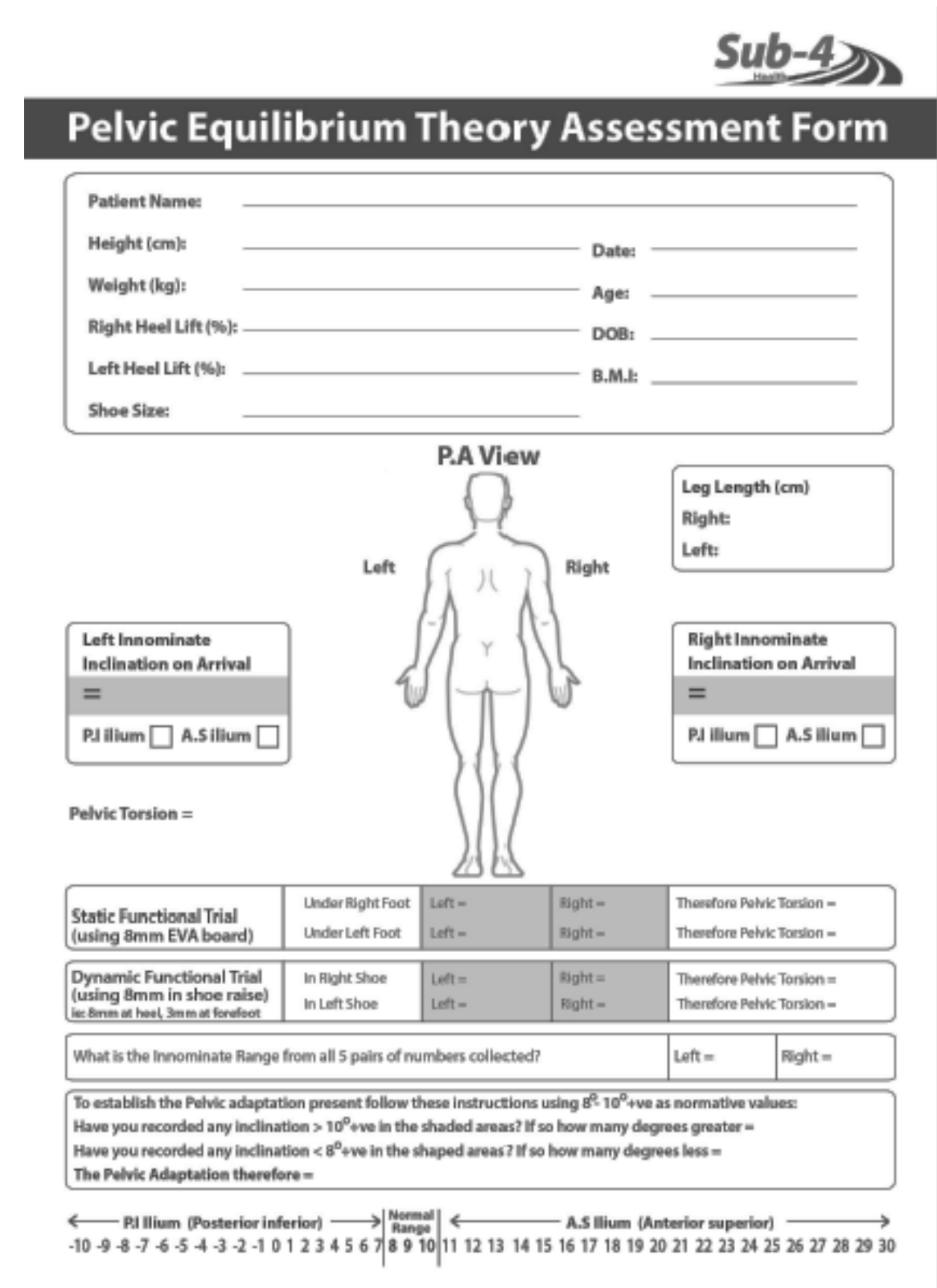

Figure 2. Pelvic Equilibrium Theory Assesment form

\section{SUBJECT}

In our preliminary research we applied the DPI in 25 children from the beginning classes (from I to III classes) in the school "Iordan Iovkov" - Sofia during the period April - May 2016. The average age was 8.6 years (from 7 to 9). Was measured left and right pelvic inclination and therefore - torsion.

\section{RESULTS}

Twenty-five children were assessed by the DPI. The results are presented in Table 2.

Table 2. Results from inclination measurement

\begin{tabular}{|l|l|}
\hline Average- left side & $10.78^{\circ}$ \\
\hline Average- right side & $10.28^{\circ}$ \\
\hline Average of Torsion & $2.108^{\circ}$ \\
\hline
\end{tabular}


The results show that the pelvic inclination in children are average for left $10.78^{\circ}$ and for right $10.28^{\circ}$. Torsion is the difference between left and right inclination of innominate bones. Mean value of torsion is $2.108^{\circ}$ this indicate potential for developing progression Lordosis in children it 1 to 3 class.

\section{DISCUSSION}

During normal standing the head is upright and facing forwards, the neck is slightly extended, the upper back is in slight flexion, and the lower back is in slight extension. The slightly extended inward curve of the neck (cervical spine) and lower back (lumbar spine) is referred to as lordosis. There are several researches about lordosis and the concomitant dysfunctions. The screening examination involved 83 children with the average age from II and III classes at the School at Sofia in October - November 2012 shows result from hamstring length test (10). In 25,30\% (21 children) of the group was indicated lordosis. The hamstring was shortened in $20(95 \%)$ of them with average $61,1^{\circ}$ (minimal value $45^{\circ}$ to maximal $75^{\circ}$ ). Only 20 children were free of spinal deformities. Their average hip flexion was $75^{\circ}$ (minimal value $60^{\circ}$ to maximal $90^{\circ}$ ).

The rate for adults is $8+v e+10^{\circ}+v e$. In the same time there are no standards for children about measuring of the pelvis inclination by DPI (6).

Our results show averages slightly above normal for pelvic inclination (Left 10.78, right 10.28).

A basic pelvic torsion measurement will clearly identify whether there is a twist (torsion) between each innominate bone, which can be quantified and recorded prior to intervention. If a pelvic torsion is identified and the physiotherapists implements their preferred treatment program to reduce the torsion, the DPI can then be used again after treatment to quantify the improvement / correction.

The functional diagnostic of the postural disorders in children plays the important role regarding the prevention of the structural spine deformities and chronic muscle skeletal problems.

\section{CONCLUSION}

This new and innovative methodology allows asymmetry, dysfunction and adaption to be quantified, allowing instant evidence based diagnosis and treatment. The DPI subsequently
TASHEVA R., et al. allows the physiotherapist to record normative values after treatment interventions have been implemented. In short this means that:

- Dysfunction can be quantified.

-Treatment becomes more directive and accurate.

- Improvement can be quantified.

- The effects of orthotics, heel-raises and other treatments can be quantified.

All these indices lead to more precise physiotherapy for prevention and correction.
Abbreviations:
DPI - Digital Pelvic Incllinometer
ASIS - anterior superior iliac spine
PSIS - posterior superior iliac spine
LLA - leg length inequality

\section{REFERENCES}

1. Beardsley, C., Egerton, T., Skinner, B., Test-re-test reliability and inter-rater reliability of a digital pelvic inclinometer in young, healthy males and females. Peer J 4, 2016.

2. Crowell, R., Cummings, G., Walker, J., Tillman, L., Intratester and intertester reliability and validity of measures of innominate bone inclination. Journal of Orthopaedic \& amp; Sports Physical Therapy, 20(2):88-97, 1994.

3. Fourchet, F., Materne, O., Rajeb, A., Horobeanu, C., Farooq, A., Pelvic tilt: reliability of measuring the standing position and range of motion in adolescent athletes. British Journal of Sports Medicine, 48(7):594-594, 2014.

4. Gnat, R., Saulicz, E., Biały, M., Kłaptocz, P., Does pelvic asymmetry always mean pathology? Analysis of mechanical factors leading to the asymmetry. Journal of Human Kinetics, 21:23-32, 2009.

5. Hagins, M., Brown, M., Cook, C., Gstalder, K., Kam, M., Kominer, G., Strimbeck, K., Intratester and intertester reliability of the palpation meter (PALM) in measuring pelvic position. Journal of Manual \& amp; Manipulative Therapy, 6(3):130-136, 1998.

6. Kendall, F., McCreary, E., et al., \& quot; Muscles, Testing and Function with Posture and Pain \& quot; Lippincott, 2005.

7. Krawiec, C., Denegar, C., Hertel, J., Salvaterra, G., Buckley, W., Static innominate asymmetry and leg length discrepancy in asymptomatic collegiate athletes. Manual Therapy, 8(4):207-213, 2003.

8. Lee, J., Yoo, W., Gak, H., The immediate effect of anterior pelvic tilt taping on pelvic 
inclination. Journal of Physical Therapy Science, 23(2):201-203, 2011.

9. McGraw, K., Wong, S., Forming inferences about some intraclass correlation coefficients. Psychological Methods, 1(1):30-46, 1996.

10.Tasheva, R., Kolev, K., Detailed Functional Diagnostic in Lumbar Lordosis Through Hamstring Length Test. 9 th FIEP European Congress 7 th International Scientific Congress "Sport, Stress, Adaptation"
TASHEVA R., et al. Physical education and Sport Competences for Life. Books of abstracts, Journal sports and science, extra issue, ISSN 1310-3393, 4, NSA "Vassil Levski", Sofia. pp 48-49, 2014.

11.Walker, M, Rothstein. J., Finucane, S., Lamb, R., Relationships between lumbar lordosis, pelvic tilt, and abdominal muscle performance. Physical Therapy 67(4):512516, 1987. 